\title{
Utility of Plasma Circulating mRNA as a Marker to Detect Hepatic Injury
}

\author{
Yuka KUDO $^{1) *}$, Takashi OCHI ${ }^{1) *}$, Hideaki SHIMADA ${ }^{1) *}$, Shinji OGAWA ${ }^{1) *}$ and Katsuhiro SHINJO ${ }^{1) * *}$ \\ ${ }^{1)}$ Discovery Biology 3, Discovery Biology Research, Pfizer Global Research and Development Nagoya Laboratories, Pfizer Japan Inc., \\ 5-2 Taketoyo, Aichi 470-2393, Japan
}

(Received 18 January 2008/Accepted 12 May 2008)

\begin{abstract}
Utility of plasma circulating mRNA as a molecular marker to detect hepatic injury was evaluated. Total RNA was isolated from plasma of the rat liver fibrosis models at various time points, and plasma circulating mRNAs of major liver-derived genes, albumin and haptoglobin, were measured by real-time quantitative reverse transcription polymerase chain reaction (qRT-PCR). Sensitivity and kinetics of plasma circulating mRNA were compared with those of plasma alanine aminotransferase (ALT) activity. We have found that the measurement of plasma circulating mRNA is more sensitive than plasma ALT activity, and enables early detection of hepatic injury. The plasma circulating mRNA will serve as a novel and highly sensitive molecular marker for hepatic injury. KEY WORDS: alanine aminotransferase, hepatic injury, plasma circulating mRNA.
\end{abstract}

J. Vet. Med. Sci. 70(9): 993-995, 2008

The existence of extracellular mRNA in the circulation, i.e., plasma and other body fluids has been long known [14]. The extracellular mRNA is thought to be released into the circulation from intact and viable cells as well as necrotic cells, and make complexes with other molecules to protect itself from RNase [3]. In normal human plasma, circulating mRNA can be detected at concentrations around $1 \mu \mathrm{g} / \mathrm{m} l$ [5]. The biological roles of circulating mRNA are still unclear, although its physiological significance has been investigated during the last several years [14]. Recently, the plasma circulating mRNA in cancer patients [7] and pregnant women [8] has been detected and analyzed with respect to sensitivity and specificity. These studies successfully demonstrated the clinical values of the detection for early cancer diagnosis and monitoring, and prenatal diagnosis of abnormal development.

Over the last decade in pharmaceutical industry, attrition of clinical compounds during the drug development process has been a big issue, in which liver toxicity is one of the leading causes [10]. In the clinic, detection and monitoring of hepatic injury or dysfunction of patients infected with hepatitis $\mathrm{B}$ and $\mathrm{C}$ are increasingly becoming important. Currently plasma aminotransferases such as ALT and aspartate aminotransferase (AST) are conventionally used to assess hepatic injury, however, it is well known that they lack specificity and sensitivity, and their levels vary in different populations and at different time points in an individual [4]. Reliable and sensitive molecular markers have been

\footnotetext{
* Present address: Kudo, Y., Free Radical Research Institute, Otsuka Pharmaceuticals Co., Ltd., Tokushima 771-0192, Japan. SHIMADA, H. and OGAWA, S., Pfizer Global Research and Development St. Louis Laboratories, 700 Chesterfield Parkway, MO 63017, U.S.A.

OCHI, T., Biomedical Research Laboratories, Takeda Pharmaceuticals Co., Ltd., Osaka 532-8686, Japan.

**Correspondence to: Shinjo, K., Central Research Laboratories, Seikagaku Corporation, Higashiyamato, Tokyo 207-0021, Japan.

e-mail: katsuhiro.shinjo@seikagaku.co.jp
}

awaited. In this study, we aimed to measure the level of plasma circulating mRNA released from damaged hepatocytes by real-time qRT-PCR, and compare its sensitivity and kinetics with that of plasma ALT to assess the potential as a molecular marker for hepatic injury.

Male Sprague-Dawley (SD) rats (200-230 g) were purchased from Japan SLC Inc. (Hamamatsu, Japan) and used in this study. All animal experiments were performed under approved protocols by the Pfizer Global Research and Development Institutional Animal Care and Use Committees. Two experimental liver fibrosis models, bile duct ligation (BDL) model $[2,6]$ and porcine serum (PS)-induced fibrosis model $[1,9,13]$, were used in this study. BDL causes cholestasis and induces acute periductular inflammation, hepatic damage and fibrosis within 14 days [2, 6]. BDL operation was performed as previously described [6]. PS injection causes chronic liver fibrosis without apparent hepatic damage and inflammatory cell infiltration in 9 weeks $[1,9,13]$. In the PS-induced fibrosis model, rats were injected with $0.5 \mathrm{ml} /$ rat of PS (COSMO BIO Co., Ltd., Tokyo, Japan) intraperitoneally (i.p.) twice a week for 9 weeks. Animals were sacrificed, and plasma and liver samples were collected on day 3, 7 and 14 after BDL and at week 2, 4, 6 and 9 after PS injection. Plasma samples were collected into syringes containing EDTA-2Na from caudal vena cava, and the supernatants were immediately used for subsequent biochemical and molecular biological analyses. A portion of liver tissue was taken from left lobe of the liver and stored at $-70^{\circ} \mathrm{C}$ until use. Total RNA was isolated from the plasma supernatant using QIAamp MinElute Virus Vacuum kit (Qiagen, Valencia, CA, U.S.A.) and treated with DNase using TURBO-DNA-free kit (Ambion Inc., Austin, TX, U.S.A.) according to manufacturer's instructions. Aliquots of total RNA were reverse transcribed into cDNA with SuperScript III ${ }^{\mathrm{TM}}$ First-Strand Synthesis System (Invitrogen Corp., Carlsbad, CA, U.S.A.). PCR TaqMan probes for rat albumin (NM_134326) and rat haptoglobin (NM_012582) and rat beta-actin (NM_031144) were pur- 
chased from Applied Biosystems (Foster City, CA, U.S.A.), and the real-time qRT-PCR was set up with Platinum Quantitative PCR SuperMix-UDG with ROX (Invitrogen Corp.) in a total volume of $20 \mu \mathrm{l}$. The amplification was monitored and analyzed on ABI PRISM ${ }^{\circledR} 7700$ Sequence Detector (Applied Biosystems) according to manufacturer's instructions. Fold increases of plasma albumin and haptoglobin mRNA of BDL or PS-treated groups over control groups were calculated using their $\mathrm{Ct}$ values by normalizing to those of plasma beta-actin mRNA. Activities of plasma ALT were determined using Transaminase CII Test Wako (Wako, Osaka, Japan) and an automatic analyzer JCABM2250 (JOEL, Tokyo, Japan). To estimate the progression of liver fibrosis, liver hydroxyproline contents were measured as described previously [11]. Statistical differences between treated groups and sham control groups were

A

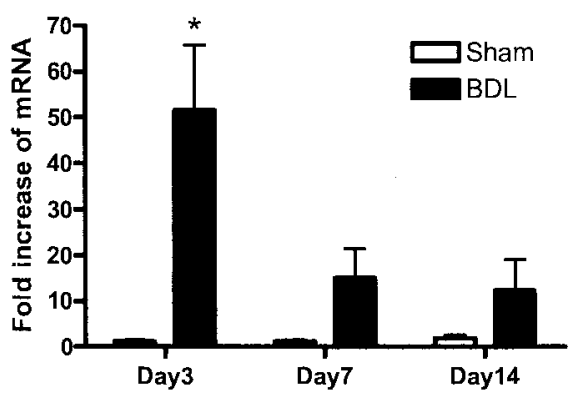

C

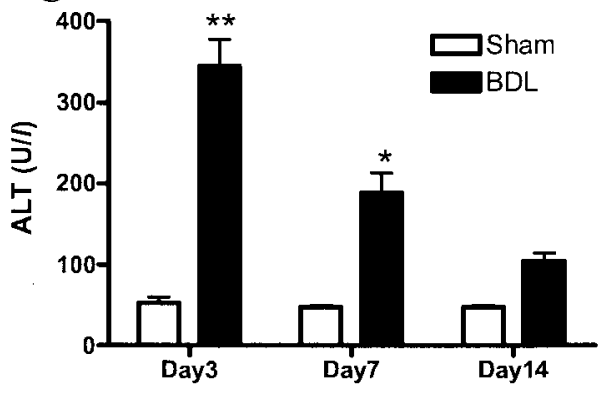

E

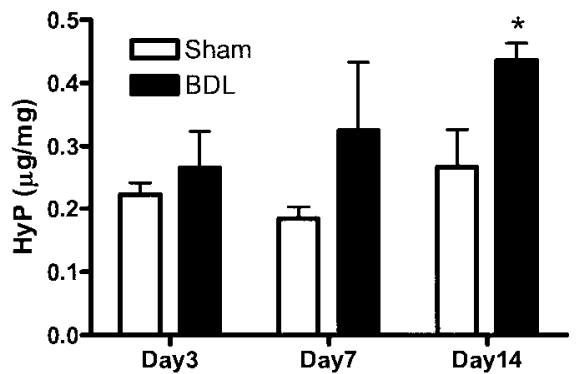

analyzed by Welch's $t$ test using the GraphPad Prism ${ }^{\circledR}$ analysis software package (GraphPad Software Inc., San Diego, CA, U.S.A.). A $P$ value $<0.05$ was considered statistically significant.

In the BDL group, some animals died before day 14 , therefore they were excluded from analyses. Activities of plasma ALT of BDL rats were elevated 6.5-fold over sham control rats and reached approximately $350 \mathrm{U} / l$ on day 3 $(P<0.001)$ followed by increases in liver hydroxyproline on day 7 and day 14 (Fig. 1C and E). The plasma albumin mRNA of BDL rats showed its peak on day 3 after operation with a $\mathrm{Ct}$ value of $31.56 \pm 1.32$ (mean $\pm \mathrm{SD}$, a representative value of three independent experiments), which was approximately 50-fold greater than that of sham control rats $(P<0.05$, Fig. 1A). The level of plasma albumin mRNA subsequently decreased on day 7 and day 14 approximately

B

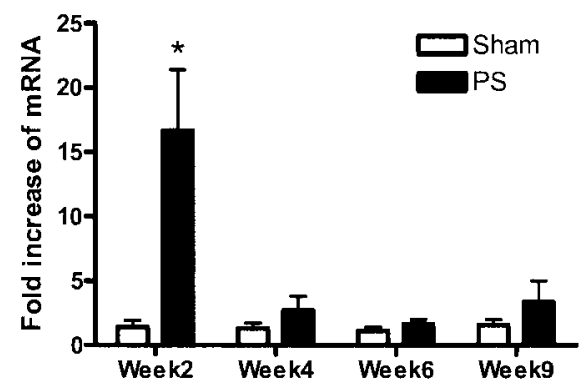

D

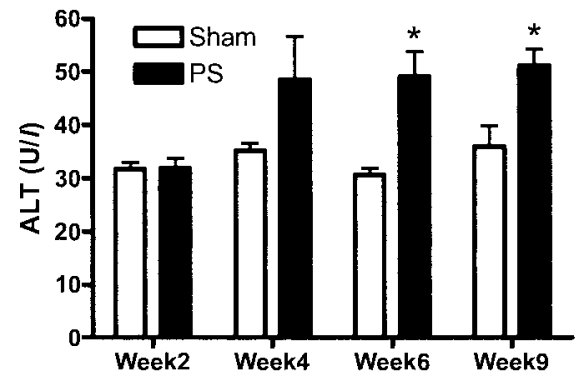

F

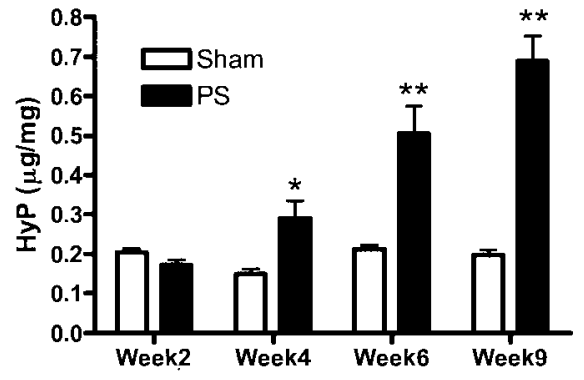

Fig. 1. Parameters in the rat BDL (A, C, E) and PS (B, D, F) models. Fold increases of plasma albumin mRNA (A, B), plasma ALT activities (U/l, C, D) and amounts of liver hydroxyproline $(\mu \mathrm{g} / \mathrm{mg}, \mathrm{E}, \mathrm{F})$ are shown. Representative data of three independent experiments are shown. Data are shown as mean \pm SD $(\mathrm{n}=3-6)$. Results were analyzed by Welch's $t$-test. * and $* *: P<0.05$ and $P<0.01$, compared with sham controls at the same time point, respectively. 
by $70 \%$ and $80 \%$, respectively. The kinetics of plasma albumin mRNA was similar to that of plasma ALT activities (Fig. 1A and C), but much larger assay windows were obtained with the former assay. Increases in plasma haptoglobin mRNA were also observed in BDL rats, but they were not as marked as plasma albumin mRNA (data not shown).

In PS-treated rats, a small but significant elevation of ALT activity (approximately $50 \mathrm{U} / 1$ ) over saline control rats started to be observed at week 6 after PS injection accompanied by an increase in liver hydroxyproline, which was characteristic of the PS-induced fibrosis model (Fig. 1D and F). However, a significant 16-fold increase in plasma albumin mRNA of PS-treated rats over saline control rats was detected at week $2(P<0.05$, Fig. 1B). This result clearly indicates that the measurement of plasma albumin mRNA is more sensitive than that of plasma ALT activity regarding the detection of hepatic injury in this chronic and mild liver fibrosis model. Significant increases in haptoglobin mRNA were not detected in PS rats throughout the experiment (data not shown).

Since animal models used here are fibrosis models, it will be of interest to investigate if plasma circulating mRNAs of fibrosis-relevant proteins can be detected. In case that any mRNA is detected either earlier or with higher sensitivity than liver hydroxyproline and plasma aminotransferases, it may serve as a non-invasive liver fibrosis marker, which will greatly impact on diagnosis and monitoring of liver fibrosis and cirrhosis patients [12].

In summary, we have shown that plasma circulating albumin mRNA may serve as a molecular marker for hepatic injury using the acute and chronic rat liver fibrosis models. The measurement of plasma circulating albumin mRNA enabled sensitive and early detection of hepatic injury with a large assay window compared with that of plasma ALT activity. With more detailed biochemical analyses and intensive proof-of-concept studies, this measurement will be developed as a powerful tool to manage liver function, and will benefit human and animal health.

ACKNOWLEDGMENT. We would like to thank Tomomi Oida for her technical assistance in the preparation for liver fibrosis models.

\section{REFERENCES}

1. Baba, Y., Uetsuka, K., Nakayama, H. and Doi, K. 2003. Rat strain differences in the early stage of porcine-serum-induced hepatic fibrosis. Exp. Toxic. Pathol. 55: 325-330.
2. Dooley, S., Hamzavi, J., Brietkopf, K., Wiercinska, E., Said, H.M., Lorenzen, J., Duke, P.T. and Gressner, A.M. 2003. Smad7 prevents activation of hepatic stellate cells and liver fibrosis in rats. Gastroenterology 125: 178-191.

3. Fleischacker, M. 2006. Biology of circulating mRNA: Still questions than answers? Ann. New York Acad. Sci. 1075: 4049.

4. Janssen, G. M. E., Kupipers, H., Willems, G. M., Does, R. J. M. M., Janssen, M. P. E. and Geurten, P. 1989. Quantification of skeletal muscle damage and relationship with metabolic variables. J. Int. Sports. Med. 10: S123-S128.

5. Kamm, R. C. and Smith, A. G. 1972. Nucleic acid concentrations in normal human plasma. Clin. Chem. 18: 519-522.

6. Kurikawa, N., Suga, M., Kuroda, S., Yamada, K. and Ishikawa, H. 2003. An angiotensin II type 1 receptor antagonist, olmesartan medoxomil, improves experimental liver fibrosis by suppression of proliferation and collagen synthesis in activated hepatic stellate cells. Br. J. Pharmacol. 139: 1085-1094.

7. Miura, N., Maeda, Y., Kanbe, T., Yazama, H., Takeda, Y., Sato, R., Tsukamoto, T., Sato, S., Marumono, A., Harada, T., Sano, A., Kishimoto, Y., Hirooka, Y., Murawaki, Y., Hasagawa, J. and Shiota, G. 2005. Serum human telomerase reverse transcriptase messenger RNA as a novel tumor marker for hepatocellular carcinoma. Clin. Cancer. Res. 11: 3205-3209.

8. Ng, E. K. O., Tsui, N. B. Y., Lau, T. K., Leung, T. N., Chiu, R. W. K., Panesar, N. S., Lit, L. C. W., Chan, K. W. and Lo, Y. M. D. 2003. mRNA of placental origin is readily detectable in maternal plasma. Proc. Natl. Acad. Sci. U.S.A. 100: 47484753.

9. Okuno, M., Akita, K., Moriwaki, H., Kawada, N., Ikeda, K., Kaneda, K., Suzuki, Y. and Kojima, S. 2001. Prevention of rat hepatic fibrosis by the protease inhibitor, camostat mesilate, ca reduced generation of active TGF- $\beta$. Gastroenterology $\mathbf{1 2 0}$ : $1784-1800$.

10. Olson, H., Betton, G., Robinson, D., Thomas, K., Monro, A., Kolaja, G., Lilly, P., Sanders, J., Sipes, G., Bracken, W., Dorato, M., Deun, K., Smith, P., Berger, B. and Heller, A. 2000. Concordance of toxicity of pharmaceuticals in humans and in animals. Regul. Toxicol. Pharmacol. 32: 56-67.

11. Reddy, G. K. and Enwemeka, C. S. 1996. A simplified method for the analysis of hydroxyproline in biological tissues. Clin. Biochem. 29: 225-229.

12. Rockey, D. C. and Bissell, D. M. 2006. Noninvasive measures of liver fibrosis. Hepatology 43: S113-S120.

13. Sakaida, I., Uchida, K., Matsumura, Y. and Okita, K. 1998. Interferon gamma treatment prevents procollagen gene expression without affecting transforming growth factor- $\beta_{1}$ expression in pig serum-induced rat liver fibrosis in vivo. J. Hepatol. 28: $471-479$.

14. Swaminathan, R. and Butt, A. N. 2006. Circulating nucleic acids in plasma and serum: Recent development. Ann. New York Acad. Sci. 1075: 1-9. 